\title{
The normoglycemic first-degree relatives of patients with type 2 diabetes mellitus have low circulating omentin- 1 and adiponectin levels
}

\author{
Samad Akbarzadeh ${ }^{\text {a }}$, Iraj Nabipour ${ }^{\mathrm{a}, *}$, Majid Assadi ${ }^{\mathrm{b}}$, Ali Movahed ${ }^{\mathrm{a}}$, Seyed Mojtaba Jafari ${ }^{\mathrm{a}}$, \\ Niloofar Motamed ${ }^{\mathrm{a}}$, Habibollah Nazem ${ }^{\mathrm{c}}$, Mehrnoosh Haraghy ${ }^{\mathrm{a}}$, Abdolreza Pourbehi ${ }^{\mathrm{c}}$, Afshar Bargahi ${ }^{\mathrm{d}}$, \\ Najmeh Hajian $^{\mathrm{a}}$ \\ ${ }^{a}$ Department of Endocrine and Metabolic Diseases, The Persian Gulf Tropical Medicine Research Center, Bushehr University of Medical Sciences, Bushehr 7514763448, Iran \\ ${ }^{b}$ Department of Hormones, The Persian Gulf Nuclear Medicine Research Center, Bushehr University of Medical Sciences, Bushehr 7514763448, Iran \\ ${ }^{\mathrm{c}}$ Department of Biochemistry, Tehran Payame Noor University, Tehran 7514763448, Iran \\ ${ }^{\mathrm{d}}$ Department of Biochemistry, The Persian Gulf Marine Biotechnology Research Center, Bushehr University of Medical Sciences, Bushehr 7514763448, Iran
}

\section{A R T I C L E I N F O}

\section{Article history:}

Received 4 July 2011

Received in revised form 28 November 2011

Accepted 7 February 2012

Available online 6 March 2012

\section{Keywords:}

Diabetes mellitus

Adipocytokines

Omentin-1

Adiponectin

Retinol-binding protein 4

\begin{abstract}
A B S T R A C T
Objective: It has been suggested that adipose-derived cytokines act as insulin sensitizers/insulin-mimetics and some others may induce insulin resistance. In order to elucidate the potential role of novel adipocytokines in the pre-diabetes states, circulating levels of novel adipocytokines were evaluated in first-degree relatives of subjects with type 2 diabetes mellitus (FDRs).

Method: Serum omentin-1, adiponectin and retinol-binding protein 4 (RBP4) levels were measured in 179 subjects (90 glucose tolerant FDRs and 89 age- and sex-matched healthy controls) using enzymelinked immunosorbent assay (ELISA) methods.

Results: There was no significant difference between the two groups regarding serum RBP4 concentrations. However, serum omentin-1 (median [interquartile range], 6.18 [4.06-11.52] ng/ml versus 10.50 [4.30$20.60] \mathrm{ng} / \mathrm{ml}, \quad p=0.004$ ) and adiponectin (mean $\pm \mathrm{SD}, 10.07 \pm 4.0 \mu \mathrm{g} / \mathrm{ml}$ versus $20.66 \pm 8.12 \mu \mathrm{g} / \mathrm{ml}$, $p<0.0001$ ) levels were significantly lower in FDRs when compared with the controls. In multiple logistic regression analysis, FDRs showed a significant association with lower circulating omentin-1 and adiponectin levels, even after adjustments were made for age, sex, body mass index, blood pressure measures, and biochemical parameters including glucose status, lipid profile, insulin levels and HOMA-IR (OR $=0.49, \mathrm{CI}$ [0.30-0.79]; $p=0.004$ and $\mathrm{OR}=0.74, \mathrm{CI}[0.67-0.82] ; p<0.0001$, respectively). However, FDRs did not show a significant association with serum RBP4 levels in different models of regression analyses.

Conclusions: The FDRs showed significant associations with lower omentin-1 and adiponectin levels. A potential role for these adipokines in the FDRs' increased risk of diabetes needs to be further elucidated.
\end{abstract}

(c) 2012 Elsevier Ltd. All rights reserved.

\section{Introduction}

It has been suggested that adipose-derived cytokines act as a potential link between energy homeostasis, immunity, neuroendocrine, atherosclerosis, type 2 diabetes, and insulin resistance [1-4]. Several of these bioactive mediators that are collectively called 'adipocytokines' may be considered insulin sensitizers/insulinmimetics, and some others may induce insulin resistance [5].

Abbreviations: FDRs, first-degree relatives of subjects with type 2 diabetes mellitus; RBP4, retinol-binding protein 4; ELISA, enzyme-linked immunosorbent assay; BMI, body mass index; OGTT, oral glucose tolerance test; HDL, high-density lipoprotein; LDL, low-density lipoprotein; HOMA-IR, homeostasis model of assessment index; $\mathrm{CV}$, coefficient of variance; WHR, waist to hip ratio.

* Corresponding author. Address: The Persian Gulf Tropical Medicine Research Center, Boostan 19 Alley, Imam Khomeini St., Bushehr, Iran. Tel.: +98 7712541827; fax: +987712541828 .

E-mail address: inabipour@gmail.com (I. Nabipour).
Omentin-1 is a novel $34 \mathrm{kDa}$ adipokine that is highly and selectively expressed in visceral adipose tissue compared with subcutaneous adipose tissue [6,7]. Furthermore, omentin-1 enhances insulin action and Akt phosphorylation [7]; it is inversely related to obesity [8] and is down regulated by insulin and glucose [9].

Adiponectin is the most abundant adipocytokine with insulin sensitizing, anti-inflammatory, and anti-atherogenic effects $[1,4]$. Adiponectin levels have inverse correlations with dyslipidemia, atherosclerosis, glucose intolerance, and insulin resistance in diverse patient populations [1,3]. In fact, hypoadiponectinemia has been suggested as one of the most important adipocytokines in the pathogenesis of type 2 diabetes [1].

The serum levels of retinol-binding protein 4 (RBP-4) reflect the risks of impaired glucose tolerance and type 2 diabetes [10]. The link between elevated RBP-4 levels and impaired insulin secretion has been shown in insulin resistant humans with obesity, impaired glucose tolerance and type 2 diabetes, and even in lean 
normoglycemic subjects with a strong family history of type 2 diabetes $[3,4,11,12]$.

Although adipokines may be involved in the pathogenesis of diabetes mellitus and insulin resistance, the contributions of individual adipokines to the pathophysiological features of insulin resistance and/or insulin sensitivity must still be fully clarified.

First-degree relatives of subjects with type 2 diabetes mellitus (FDRs) frequently have insulin resistance [13]. The results of a large population study [14] showed that $\beta$ cell impairment exists in the offspring of type 2 diabetes patients, even in the absence of insulin resistance. Thus, $\beta$ cell dysfunction is the outstanding determinant for the development of diabetes mellitus in this group.

FDRs bear a 40\% lifetime risk for developing type 2 diabetes mellitus [15]. The background of insulin resistance/ $\beta$ cell dysfunction and glucose metabolism in FDRs used to assess the circulating levels of novel adipocytokines such as omentin, RBP-4 and adiponectin may be helpful in illustrating some pathophysiological aspects of adipose tissue involvement in the development of insulin resistance in this group. In the present study, we performed the first evaluation of serum omentin in addition to assessing RBP4 and adiponectin levels in FDRs, matching control subjects without a family history of type 2 diabetes mellitus.

\section{Methods}

\subsection{Subjects and physical measurements}

Ninety normoglycemic subjects (mean age \pm SD $40.55 \pm$ 9.36 years: 41 men and 49 women) who were first-degree relatives of patients with type 2 diabetes mellitus (FDRs) and who consecutively visited the Endocrine Clinic at Fatemeh-Zahra University Hospital were enrolled in this study. The age and sex-matched controls ( $41.375 \pm 9.47$ years: 49 men, 40 women) were selected from a cohort of healthy subjects recruited by the Persian Gulf Healthy Heart Study at Bushehr University of Medical Sciences [16] for evaluation of cardiovascular risk factors in the Persian Gulf region. The following criteria were used for the healthy controls: (1) they had no family history of diabetes mellitus, type 2 diabetes or endocrine disorders, cardiovascular diseases, or hepatic or renal dysfunction; (2) they had no history of taking antidiabetic and antiobesity drugs, glucocorticoids, anti-inflammatory agents, estrogenic or androgenic medications.

The participants in the control group had anthropometric measurements comparable to those of the FDRs (Table 1). All subjects were asked to fast and to present to the Persian Gulf Health Research Center between 7:30 and 9:30 a.m. They underwent a 75 -g oral glucose tolerance test (OGTT). The definition of glucose tolerance was a subject who had a fasting plasma glucose level lower than $110 \mathrm{mg} / \mathrm{dl}$ and a second-hour blood sugar level after oral glucose load (2 h-OGTT) lower than $140 \mathrm{mg} / \mathrm{dl}$ [17].

Blood pressure was assessed twice at the right arm after a 15 min rest in the sitting position, using a standard mercury sphygmomanometer. Height and weight were measured using a stadiometer. Heavy outer garments and shoes were removed before height and weight were measured. Body mass index (BMI) was calculated. Waist circumference was defined at the midway level between the costal margins and the iliac crests. Hip circumference was measured at the level of the greater trochanters. Waist-to-hip ratio was calculated for all participants.

The study was approved by the Medical Ethics Committee of Bushehr University of Medical Sciences.

\subsection{Laboratory measurements}

A fasting blood sample was taken, all samples were promptly centrifuged, and sera were separated and kept frozen at $-70^{\circ} \mathrm{C}$ until they were used. Analyses for biochemical parameters (blood glucose, triglyceride, and cholesterol levels) were carried out at the Persian Gulf Health Research Center on the day of blood collection using a Selectra 2 autoanalyzer (Vital Scientific, Spankeren, The Netherlands). Glucose was assayed with the enzymatic (glucose oxidase) colorimetric method using a commercial kit (Pars Azmun Inc., Tehran, Iran). Serum total cholesterol and HDL (highdensity lipoprotein) cholesterol were measured using cholesterol oxidase phenol aminoantipyrine and triglycerides using the glycerol-3 phosphate oxidase phenol aminoantipyrine enzymatic method. Serum LDL (low-density lipoprotein) cholesterol was calculated using the Friedwald formula. LDL cholesterol was not calculated when the triglycerides concentration was $>400 \mathrm{mg} / \mathrm{dl}$.

Insulin was measured using a commercially available enzymelinked immunosorbent assay kit (Insulin ELISA, DRG Diagnostics, Marburg, Germany). The assay sensitivity was $1.76 \mu \mathrm{IU} / \mathrm{ml}$; the intra- and inter-assay coefficients of variance were $1.79-2.6 \%$ and 2.88-5.99\%, respectively.

Insulin resistance was assessed by calculating the homeostasis model of assessment index (HOMA-IR) using the following equation: fasting insulin $(\mu \mathrm{IU} / \mathrm{ml}) \times$ fasting glucose $(\mathrm{mg} / \mathrm{dl}) / 405$.

Serum omentin- 1 concentrations were measured using manual omentin-1 (human) detection (ELISA kit [intelectin-1 (human) ELISA kit, Apotech Corporation, Switzerland]). The detection limit of the assay was $0.4 \mathrm{ng} / \mathrm{ml}$ (range $0.5-32 \mathrm{ng} / \mathrm{ml}$ ). The mean intraassay and inter-assay CVs of the omentin- 1 assay were $4.51-7.4 \%$ and $4.19-9.27 \%$, respectively. The antibodies used in this kit are specific to the measurement of natural and recombinant human omentin-1.

To detect adiponectin in the serum samples, commercially (Cat. No. AG-45A-0001EK-KI01) available enzyme-linked immunosorbent assay kits (AdipoGen, Incheon, Korea) were used according to the manufacturer's instructions. The limit of detection of the assay was $100 \mathrm{pg} / \mathrm{ml}$; the intra- and inter-assay coefficients of variance were $2.9-3.8 \%$ and $2.8-5.5 \%$, respectively.

For the detection of RBP4 in the serum samples, commercially available ELISA (AdipoGen, Incheon, Korea) kits were used according to the manufacturer's instructions. The assay sensitivity was

Table 1

The general characteristics, including blood pressure and anthropometric measurements, and the biochemical parameters of first-degree relatives of subjects with type 2 diabetes mellitus (FDRs) and healthy controls.

\begin{tabular}{lllc}
\hline & FDRs $(n=90)$ & Control $(n=89)$ & $p$ Value \\
\hline Female/male ratio & $49 / 41$ & $40 / 49$ & 0.233 \\
Age $($ years $)$ & $40.55 \pm 9.63$ & $41.37 \pm 9.47$ & 0.575 \\
Systolic BP $(\mathrm{mmHg})$ & $115 \pm 13.8$ & $115.82 \pm 12.84$ & 0.687 \\
Diastolic BP $(\mathrm{mmHg})$ & $74.72 \pm 9.95$ & $74.45 \pm 10.47$ & 0.864 \\
WHR & $0.90 \pm 0.14$ & $0.91 \pm 0.17$ & 0.511 \\
BMI $\left(\mathrm{kg} / \mathrm{m}^{2}\right)$ & $27.57 \pm 4.25$ & $26.52 \pm 4.24$ & 0.104 \\
Fasting glucose $(\mathrm{mg} / \mathrm{dl})$ & $80.5 \pm 8.56$ & $79.77 \pm 10.46$ & 0.612 \\
2 h-OGTT $(\mathrm{mg} / \mathrm{dl})$ & $92.17 \pm 15.68$ & $92.03 \pm 16.65$ & 0.956 \\
Total cholesterol $(\mathrm{mg} / \mathrm{dl})$ & $198.54 \pm 36.85$ & $196.92 \pm 37.24$ & 0.770 \\
Triglyceride $(\mathrm{mg} / \mathrm{dl})$ & $155.72 \pm 82.3$ & $155.52 \pm 75$ & 0.279 \\
HDL-cholesterol $(\mathrm{mg} / \mathrm{dl})$ & $43 \pm 10.38$ & $45.44 \pm 11.65$ & 0.140 \\
LDL-cholesterol $(\mathrm{mg} / \mathrm{dl})$ & $121.01 \pm 31.55$ & $120.04 \pm 32.44$ & 0.840 \\
Insulin $(\mu \mathrm{IU} / \mathrm{ml})$ & $8.75(5.25-16.68)$ & $6.56(5.03-10.18)^{*}$ & 0.027 \\
HOMA-IR & $1.82(1.05-3.06)$ & $1.29(0.98-1.93)^{*}$ & 0.010 \\
Omentin-1 $(\mathrm{ng} / \mathrm{ml})$ & $6.18(4.06-11.52)$ & $10.50(4.30-20.60)$ & 0.004 \\
RBP4 $(\mu \mathrm{mg} / \mathrm{ml})$ & $100.0 \pm 42.73$ & $95.28 \pm 55.34$ & 0.535 \\
Adiponectin $(\mu \mathrm{g} / \mathrm{ml})$ & $10.07 \pm 4.0$ & $20.66 \pm 8.12$ & $<0.0001$
\end{tabular}

BMI, body mass index; WHR, waist to hip ratio; BP, blood pressure; 2 h-OGTT, second-hour oral glucose tolerance test; HOMA-IR, homeostasis model of assessment index; RBP4, retinol-binding protein 4.

Data are means (SD), except for insulin and HOMA-IR, and omentin-1 which are medians (interquartile ranges).

$p$ values $<0.05$ (the FDRs in comparison to the controls). 
$380 \mathrm{pg} / \mathrm{ml}$; the intra- and inter-assay coefficients of variance were $1.7-3.7 \%$ and $5.9-8.8 \%$, respectively.

\subsection{Statistical analysis}

Normal distribution of the data was controlled with the Kolmogorov-Smirnov test. Probability values $<5 \%$ were considered statistically significant. The significance of the difference in the results between the two groups was determined with chi-square analysis using $2 \times 2$ contingency tables. A two-tailed $t$-test was used to compare the values across groups in the presence of a normal distribution. Significant differences were assessed with the MannWhitney $U$ test in the absence of a normal distribution. Analysis of variance (ANOVA) was performed to test differences in adipokines across quartiles of measures of obesity and HOMA-IR.

Because the distributions of serum insulin levels, HOMA-IR and omentin-1 were skewed, logarithmically transformed values for HOMA-IR and natural logarithmically transformed values for omentin-1 were used for statistical analysis.

Multiple linear regression models were used to assess age- and sex-adjusted associations of serum omentin-I, adiponectin and RBP4 levels in relation to anthropometric and blood pressure measurements as well as biochemical variables in both the FDRs and the healthy controls.

Binary logistic regression analysis was used to ascertain the associations between the presence of FDRs and circulating adipocytokines (omentin-1, adiponectin, and RBP4) levels. In the full model, the presence of FDRs versus healthy controls was the dependent variable. Age, sex, BMI, systolic and diastolic blood pressures, HOMA-IR, fasting glucose, second-hour blood glucose, insulin, triglyceride, HDL-cholesterol and LDL-cholesterol levels, and circulating adipocytokines were considered covariates.

All statistical analyses were performed using the PASW Statistics GradPack 18 (SPSS Inc., Chicago, IL).

\section{Results}

The characteristics of the study participants are shown in Table 1 . There were no significant differences in age, gender, blood pressure measurements, height, weight, waist and hip circumferences, waist to hip ratio, and BMI between the FDRs and the controls. There was also no significant difference in the fasting blood glucose, 2 h-OGTT, and lipid profiles between the two groups. However, the FDRs had higher HOMA-IR and serum insulin levels than the control group ( $p<0.05$, Table 1$)$.

The serum levels of the studied adipocytokines of the FDRs and the healthy controls are also shown in Table 1 . There was no significant difference between the two groups regarding serum RBP4 concentrations. However, serum omentin-1 (median [interquartile range], 6.18 [4.06$11.52] \mathrm{ng} / \mathrm{ml}$ versus 10.50 [4.30-20.60] ng/ml, $p=0.004$ ) and adiponectin levels (mean $\pm \mathrm{SD}, 10.07 \pm 4.0 \mu \mathrm{g} / \mathrm{ml}$ versus $20.66 \pm 8.12 \mu \mathrm{g}$ / $\mathrm{ml}, p<0.0001$ ) were significantly lower in the FDRs when compared with the controls. No gender difference was found for the circulating omentin- 1 , adiponectin and RBP4 levels $(p>0.05)$.

Age- and sex-adjusted serum omentin-1, adiponectin and RBP4 levels had no correlations with anthropometric and blood pressure measurements, fasting blood glucose, 2 h-OGTT, triglyceride, total cholesterol, HDL-cholesterol, LDL-cholesterol, insulin, and HOMAIR in the FDRs and the healthy controls ( $p>0.05$, data not shown). Furthermore, the age- and sex-adjusted adipokines serum levels showed no linear correlations with each other.

The studied population was divided into quartiles of BMI, WHR, and HOMA-IR. There were no significant differences across quartiles of BMI for omentin-1, adiponectin, and RBP4 levels. The results did not change after stratifying the studied groups by sex.
Table 2

Unadjusted and adjusted odds ratios [OR; 95\% confidence interval $(\mathrm{CI})$ ] between the presence of first-degree relatives of subjects with type 2 diabetes mellitus (FDRs) and circulating adipocytokines (omentin-1, RBP4, and adiponectin) levels.

\begin{tabular}{llll}
\hline & OR & CI & $p$ Value \\
\hline & Omentin-1 & & \\
Unadjusted & 0.50 & $0.33-0.75$ & 0.001 \\
Adjusted for age + sex & 0.49 & $0.32-0.74$ & 0.001 \\
Adjusted for age + sex + BMI & 0.50 & $0.33-0.77$ & 0.001 \\
Adjusted for age + sex + WHR & 0.49 & $0.32-0.79$ & 0.001 \\
Full model & 0.49 & $0.30-0.79$ & 0.004 \\
& RBP4 & & \\
Unadjusted & 1.0 & $0.99-1.0$ & 0.533 \\
Adjusted for age + sex & 1.0 & $0.99-1.0$ & 0.395 \\
Adjusted for age + sex + BMI & 1.0 & $0.99-1.0$ & 0.450 \\
Adjusted for age + sex + WHR & 1.0 & $0.99-1.0$ & 0.435 \\
Full model & 1.0 & $0.99-1.01$ & 0.561 \\
& Adiponectin & & \\
Unadjusted & 0.77 & $0.71-0.83$ & $<0.0001$ \\
Adjusted for age + sex & 0.75 & $0.69-0.82$ & $<0.0001$ \\
Adjusted for age + sex + BMI & 0.75 & $0.69-0.83$ & $<0.0001$ \\
Adjusted for age + sex + WHR & 0.76 & $0.69-0.82$ & $<0.0001$ \\
Full model & 0.74 & $0.67-0.82$ & $<0.0001$ \\
\hline
\end{tabular}

BMI, body mass index; WHR, waist to hip ratio.

Full model included age, sex, BMI, systolic and diastolic blood pressures, HOMA-IR, fasting glucose, second-hour blood glucose, insulin, triglyceride, HDL-cholesterol, and LDL-cholesterol levels.

There were no significant differences across quartiles of WHR for omentin-1, and adiponectin levels. However, circulating RBP4 showed a significant difference across WHR quartiles $(p=0.033)$. Compared with subjects in the lowest quartile of WHR, those in the highest quartile had a significantly higher means of RBP4 $(111.61 \pm 49.27 \mu \mathrm{g} / \mathrm{ml}$ versus $79.88 \pm 50.52 \mu \mathrm{g} / \mathrm{ml}, p=0.007)$.

Analysis by quartiles of HOMA-IR revealed an inverse significant association with serum levels of adiponectin $(p=0.011)$. Compared with subjects in the lowest quartile of HOMA-IR, those in the highest quartile had a significantly lower means of serum adiponectin $(12.67 \pm 7.77 \mu \mathrm{g} / \mathrm{ml}$ versus $16.85 \pm 9.33 \mu \mathrm{g} / \mathrm{ml}, p=0.027)$. However, analysis by quartiles of HOMA-IR did not reveal significant associations with serum levels of RBP4 and in omentin- 1 . The results did not change after stratifying the studied groups by sex.

Table 2 shows unadjusted and adjusted odds ratios (95\% CI) between the presence of FDRs and circulating adipocytokines levels. In multiple logistic regression analysis, the FDRs showed a significant association with lower circulating omentin- 1 and adiponectin levels, even after adjustments were made for age, sex, BMI, blood pressure measures and biochemical parameters including glucose status, lipid profile, insulin levels, and HOMA-IR (OR $=0.49, \mathrm{CI}$ [0.30-0.79]; $p=0.004$ and $\mathrm{OR}=0.74$, CI [0.67-0.82]; $p<0.0001$, respectively). However, the FDRs did not show a significant association with serum RBP4 levels in different models of regression analyses (Table 2).

\section{Discussion}

In the current study, we found that the FDRs had a significant association with lower omentin-1 and adiponectin levels, even after adjusting for age, sex, BMI, and biochemical parameters in multivariate analyses. However, no significant difference was found in serum RBP4 concentrations between the FDRs and the controls.

Omentin-1 is a newly identified depot-specific adipokine in human adipose tissue that may modulate insulin sensitivity by its paracrine or endocrine functions [7]. It has been shown that omentin-1 levels were inversely correlated with measures of obesity and 
insulin resistance, and positively correlated with adiponectin levels [8]. Expression of omentin-1 mRNA and protein levels are decreased in polycystic ovary syndrome, which is associated with obesity and insulin resistance [9]. It is interesting that circulating omentin-1 concentrations increased after weight loss and with improvement of insulin sensitivity [18]. Aerobic training in overweight and obese men was accompanied by increased omentin-1 concentrations [19]. It has been reported that omentin-1 levels were lower in patients with metabolic syndrome than in the controls [20].

The results of these studies confirm that omentin-1 may contribute to the underlying pathophysiology of insulin resistance.

Impaired glucose metabolism is common in FDRs [13]. They also have defects in $\beta$ cell function when they are not hyperglycemic, and this reduction affects insulin and amylin responses to glucose stimulation [21]. Since both insulin sensitivity and $\beta$ cell dysfunction predict conversion to diabetes [14,22], FDRs are at increased risk of type 2 diabetes [15]. Therefore, they are very good available models to study the early development of insulin resistance in a pre-diabetes state.

In the current study, we observed for the first time that circulating omentin-1 levels were lower in FDRs than the controls. However, decreased omentin mRNA expression levels were reported in patients with type 2 diabetes and obesity [23]. In two recent studies, serum omentin levels were lower in patients with type 2 diabetes and impaired glucose regulation than in the normal glucose tolerance group [24,25].

In the current study, the observed reduced levels of omentin-1 may suggest a potential role for this adipocytokine in the FDRs' increased risk of type 2 diabetes.

RBP4 is a newly discovered adipokine and was suggested as a potential link with type 2 diabetes mellitus by Yang et al. [11]. They showed that serum RBP4 levels were elevated in GLUT4 glucose transporter knockout mice and humans with obesity and type 2 diabetes [11]. Currently, increasing evidence denotes an association between increased circulating levels of RBP4 with type 2 diabetes mellitus and/or insulin resistance states [11,25,12,26-31]. A pathophysiological link between RBP4 and type 2 diabetes mellitus may also arise from impaired insulin secretion [3]. By evaluation of insulin secretion, insulin sensitivity, insulin disposition index and serum RBP4 in non-diabetic men within a wide range of obesity, Broch et al. suggested that RBP4 may impact $\beta$-cell secretion [32].

Only two studies evaluated circulating RBP4 levels in FDR. In one of these studies, the authors found that RBP4 correlated inversely with the rate of glucose disposal and correlated positively with fasting insulin levels among non-obese, normoglycemic subjects with at least one first-degree relative with type 2 diabetes [12]. In the second study, serum RBP4 levels were reported to be significantly elevated in the normoglycemic FDR group compared with the control group [33]. In contrast to these studies, we found no significant difference for circulating RBP4 levels between the FDR and the control group. The discrepancies with these two studies of our obtained results cannot be explained until the results of larger studies are available. However, differences in the selection and the characteristics of the studied populations, including the use of different measuring systems, may be potential contributing factors.

Reduced adiponectin mRNA in subcutaneous adipose tissue was reported in FDRs by Lihn et al. [34]. Accordingly, they suggested that adiponectin gene expression is dysregulated in FDR. Similar to our finding, Pellme et al. reported that plasma adiponectin levels were decreased in non-obese but insulin-resistant FDR [35]. Skeletal muscle adiponectin receptors gene expression levels, as well as plasma adiponectin concentration were reported to be lower in subjects with a family history of type 2 diabetes than in those with no family history of the disease. In addition, there was a positive correlation between adiponectin receptors gene expression levels in skeletal muscles and insulin sensitivity [36]. Reduced circulating adiponectin levels were also reported in lean and normoglycemic offspring of type 2 diabetic subjects [37,38]. In a cross-sectional study among healthy Korean men and women, lower serum adiponectin levels were associated with a family history of diabetes independently of obesity and insulin resistance [39]. Recently, it has been shown that the FDR group had lower adiponectin levels versus the normal group at baseline, and these levels were reduced in both groups over a five year period [40].

Together these observations support the concept that adiponectin may play a role in the development of insulin resistance in FDR.

Although we found that there were no linear correlations between these novel adipokines and BMI or WHR, the studied subjects who had the fourth quartile of WHR showed higher RBP4 levels compared with those in the first quartile of WHR. However, the medical literature is inconsistent in the correlations between circulating adipokines and obesity measurements. In our study, the lack of strong linear correlations between the indices of obesity and these novel adipokines could be caused by the small number of participants and narrow range of adiposity measures among the studied population. Furthermore, the subjects of our study were healthy, except for the positive family history of type 2 diabetes in the FDR group. However, substantial studies about adipokines in relationship to obesity were conducted among subjects with glucose intolerance, type 2 diabetes mellitus, polycystic ovary syndrome, non-fatty liver disease or the metabolic syndrome.

Likewise, age- and sex-adjusted omentin-1, RBP4 and adipokine levels showed no correlations with HOMA-IR in the FDR and control groups. However, circulating adiponectin levels were lower in the fourth quartile of HOMA-IR than in the first quartile of HOMA-IR. Several studies have confirmed correlations between insulin sensitivity, circulating adiponectin [41,42], RBP4 [2,11], and omentin-1 [13], but these associations may be more detectable in clamp-derived indices of insulin sensitivity than HOMA-derives indices, which are less sensitive [2].

We acknowledge several study limitations. As our data are cross-sectional, limited inferences can be made regarding temporality and causation. In the current study, insulin sensitivity with the gold-standard isoglycaemic-hyperinsulinemic clamp test was not measured; however, good correlations between HOMA-IR and insulin resistance assessed by some "gold standard" methods have been reported [43]. Another limitation of our study includes the lack of assessment of $\beta$ cell function. Since we assessed the investigated adipocytokines with single measurements, the changes in these adipocytokines over time could not be reflected in the current study.

In conclusion, FDRs had significantly reduced levels of omentin1 and adiponectin levels compared with the control subjects, but they had similar circulating levels of RBP4. The observed lower levels of omentin-1 and adiponectin in the FDR group were independent of metabolic and biochemical variables. The physiologic and pathophysiologic contribution of these findings remains to be explained but may indicate a pathophysiological role for omentin-1 and adiponectin in the FDRs who bear a high lifetime risk for developing type 2 diabetes mellitus.

To clarify the role of circulating adipocytokines in relation to the worsening of glucose tolerance over time in FDRs, longitudinal studies with concurrent measurements of additional adipocytokines, inflammatory markers and cytokines would elucidate the complex system that regulates beta cell function and insulin sensitivity in pre-diabetes states.

\section{Conflict of interest}

None of the authors has the conflicting financial interest. 


\section{Acknowledgments}

This study was supported in part by a grant from Bushehr Province Technology and Research Committee and Research Deputy of Bushehr University of Medical Science.

\section{References}

[1] Gulcelik NE, Usman A, Gurlek A. Role of adipocytokines in predicting the development of diabetes and its late complications. Endocrine 2009;36: 397-403.

[2] Perseghin G, Lattuada G, De CobelliF F, et al. Serum retinol-binding protein-4, leptin, and adiponectin concentrations are related to ectopic fat accumulation. J Clin Endocrinol Metab 2007;92:4883-8.

[3] Esteve E, Ricart W, Fernandez-Real JM, et al. Adipocytokines and insulin resistance. the possible role of lipocalin-2, retinol binding protein-4, and adiponectin. Diabetes Care 2009;32:S362-7.

[4] Rabe K, Lehrke M, Parhofer KG, et al. Adipokines and insulin resistance. Mol Med 2008;14:741-51.

[5] Kralisch S, Bluher M, Paschke M, et al. Adipokines and adipocyte targets in the future management of obesity and the metabolic syndrome. Mini Rev Med Chem 2007:7:39-45.

[6] Schaffler A, Neumeier M, Herfarth H, Furst A, Scholmerich J, Buchler C. Genomic structure of human omentin, a new adipocytokine expressed in omental adipose tissue. Biochim Biophys Acta 2005;1732:96-102.

[7] Yang RZ, Lee MJ, Hu H, et al. Identification of omentin as a novel depot-specific adipokine in human adipose tissue: possible role in modulating insulin action. Am J Physiol Endocrinol Metab 2006;290:E1253-61.

[8] de Souza Batista CM, Yang RZ, Lee MJ, et al. Omentin plasma levels and gene expression are decreased in obesity. Diabetes 2007;56:1655-61.

[9] Tan BK, Adya R, Farhatullah S, et al. Omentin-1, a novel adipokine, is decreased in overweight insulin-resistant women with polycystic ovary syndrome: ex vivo and in vivo regulation of omentin-1 by insulin and glucose. Diabetes 2008;57:801-8.

[10] Cho YM, Youn BS, Lee $\mathrm{H}$, et al. Serum retinol-binding protein-4 concentrations are elevated in human subjects with impaired glucose tolerance and type 2 diabetes. Diabetes Care 2006;29:2457-61.

[11] Yang Q, Graham TE, Mody N, et al. Serum retinol binding protein 4 contributes to insulin resistance in obesity and type 2 diabetes. Nature 2005;436:356-62.

[12] Graham TE, Yang $\mathrm{Q}$, Bluher $\mathrm{M}$, et al. Retinol-binding protein 4 and insulin resistance in lean, obese, and diabetic subjects. N Engl J Med 2006;354: 2552-63.

[13] Eriksson J, Franssila-Kallunki A, Ekstrand A, Saloranta C, Widen E, Schalin C, et al. Early metabolic defects in persons at increased risk for non-insulindependent diabetes mellitus. N Engl J Med 1989;321:337-43.

[14] Stadler M, Pacini G, Petrie J, Luger A, Anderwald C. RISC Investigators. $\beta$ cell (dys)function in non-diabetic offspring of diabetic patients. Diabetologia 2009;52:2435-44

[15] Perseghin G, Ghosh S, Gerow K, Shulman GI. Metabolic defects in lean nondiabetic offspring of NIDDM parents: a cross-sectional study. Diabetes 1997;46:1001-9.

[16] Nabipour I, Amiri M, Imami SR, Jahfari SM, Shafeiae E, Nosrati A, et al. The metabolic syndrome and nonfatal ischemic heart disease; a population-based study. Int J Cardiol 2007;118:48-53.

[17] Carnevale Schianca GP, Rossi A, Sainaghi PP, Maduli E, Bartoli E. The significance of impaired fasting glucose versus impaired glucose tolerance. Importance of insulin secretion and resistance. Diabetes Care 2003;26:1333-7.

[18] Moreno-Navarrete JM, Catalan V, Ortega F, et al. Circulating omentin concentration increases after weight loss. Nutr Metab (Lond) 2010;7:27.

[19] Saremi A, Asghari M, Ghorbani A. Effects of aerobic training on serum omentin-1 and cardiometabolic risk factors in overweight and obese men. J Sports Sci 2010;28:993-8.

[20] Liu R, Wang X, Bu P. Omentin-1 is associated with carotid atherosclerosis in patients with metabolic syndrome. Diabetes Res Clin Pract 2011, Apr 15. [Epub ahead of print].

[21] Knowles NG, Landchild MA, Fujimoto WY, Kahn SE. Insulin and amylin release are both diminished in first-degree relatives of subjects with type 2 diabetes. Diabetes Care 2002;25:292-7.
[22] Warram JH, Martin BC, Krolewski AS, Soeldner JS, Kahn CR. Slow glucose removal rate and hyperinsulinemia precede the development of type II diabetes in the offspring of diabetic parents. Ann Intern Med 1990;113:909-15.

[23] Cai RC, Wei L, Di JZ, et al. Expression of omentin in adipose tissues in obese and type 2 diabetic patients. Zhonghua Yi Xue Za Zhi 2009; 89: 381-4.

[24] Pan HY, Guo L, Li Q. Changes of serum omentin-1 levels in normal subjects and in patients with impaired glucose regulation and with newly diagnosed and untreated type 2 diabetes. Diabetes Res Clin Pract 2010;88:29-33.

[25] Yan P, Liu D, Long M, Ren Y, Pang J, Li R. Changes of serum omentin levels and relationship between omentin and adiponectin concentrations in type 2 diabetes mellitus. Exp Clin Endocrinol Diabetes 2011;119:257-63.

[26] Cho YM, Youn BS, Lee H, et al. Plasma retinol-binding protein-4 concentrations are elevated in human subjects with impaired glucose tolerance and type 2 diabetes. Diabetes Care 2006;29:2457-61.

[27] An C, Wang H, Liu X, et al. Serum retinol-binding protein 4 is elevated and positively associated with insulin resistance in postmenopausal women. Endocr J 2009;56:987-96.

[28] Al-Daghri NM, Al-Attas OS, Alokail M, et al. Retinol binding protein-4 is associated with TNF- $\alpha$ and not insulin resistance in subjects with type 2 diabetes mellitus and coronary heart disease. Dis Markers 2009;26:135-40.

[29] Cheng X, Zhang H. Serum retinal-binding protein 4 is positively related to insulin resistance in Chinese subjects with type 2 diabetes. Diabetes Res Clin Pract 2009;84:58-60.

[30] Jia W, Wu H, Bao Y, et al. Association of serum retinol-binding protein 4 and visceral adiposity in Chinese subjects with and without type 2 diabetes. J Clin Endocrinol Metab 2007:92:3224-9.

[31] Takebayashi K, Suetsugu M, Wakabayashi S, et al. Retinol binding protein-4 levels and clinical features of type 2 diabetes patients. J Clin Endocrinol Metab 2007;92:2712-9.

[32] Broch M, Vendrell J, Ricart W, et al. Circulating retinol-binding protein-4 insulin sensitivity, insulin secretion, and insulin disposition index in obese and nonobese subjects. Diabetes Care 2007;30:1802-6.

[33] Gao S, Li M, Wang Z, et al. Serum levels of retinol-binding protein-4 and its association with metabolic syndrome in first-degree relatives of type 2 diabetes mellitus. Zhonghua Yi Xue Za Zhi 2009;89:2129-33.

[34] Lihn AS, Ostergard T, Nyholm B, et al. Adiponectin expression in adipose tissue is reduced in first-degree relatives of type 2 diabetic patients. Am J Physiol Endocrinol Metab 2003;284:E443-8.

[35] Pellme F, Smith U, Funahashi T, et al. Circulating adiponectin levels are reduced in nonobese but insulin-resistant first-degree relatives of type 2 diabetic patients. Diabetes 2003;52:1182-6.

[36] Civitarese AE, Jenkinson CP, Richardson D, et al. Adiponectin receptors gene expression and insulin sensitivity in non-diabetic Mexican Americans with or without a family history of type 2 diabetes. Diabetologia 2004;47:816-20.

[37] Kowalska I, Straczkowski M, Nikowska A, et al. Plasma adiponectin concentration and tumor necrosis factor-alpha system activity in lean non-diabetic offspring of type 2 diabetic subjects. Eur J Endocrinol 2006;154:319-24.

[38] Tesauro M, Rizza S, Iantorno M, et al. Vascular, metabolic, and inflammatory abnormalities in normoglycemic offspring of patients with type 2 diabetes mellitus. Metabolism 2007;56:413-9.

[39] Sull JW, Kim HJ, Yun JE, et al. Serum adiponectin is associated with family history of diabetes independently of obesity and insulin resistance in healthy Korean men and women. Eur J Endocrinol 2009;160:39-43.

[40] Liu J, Wang F, Cha Y, et al. Adiponectin levels in non-obese first-degree relatives of type 2 diabetes patients and non-diabetic subjects: a 5-year follow-up study. J Int Med Res 2010;38:792-802.

[41] Hotta K, Funahashi T, Bodkin NL, et al. Circulating concentrations of the adipocyte protein adiponectin are decreased in parallel with reduced insulin sensitivity during the progression to type 2 diabetes in rhesus monkeys. Diabetes 2001:50:1126-33.

[42] Weyer C, Funahashi T, Tanaka S, et al. Hypoadiponectinemia in obesity and type 2 diabetes: close association with insulin resistance and hyperinsulinemia. J Clin Endocrinol Metab 2001;86:1930-5.

[43] Song Y, Manson JE, Tinker L, Howard BV, Kuller LH, Nathan L, et al. Insulin sensitivity and insulin secretion determined by homeostasis model assessment and risk of diabetes in a multiethnic cohort of women: the Women's Health Initiative Observational Study. Diabetes Care 2007;30: 1747-52. 